LUX, et al.

\title{
IMPLICATIONS OF UNCERTAINTIES ON EUROPEAN DEMO DESIGN
}

H. Lux

CCFE, UKAEA

Abingdon, UK

Email: Hanni.Lux@ukaea.uk

M. Siccinio

Max-Planck-Institute for Plasma Physics and EUROfusion Programme Management Unit

Garching, Germany

W. Biel

Forschungszentrum Julich GmbH, Institut fur Energie- und Klimaforschung - Plasmaphysik

Julich, Germany

G. Federici

EUROfusion Programme Management Unit

Garching, Germany

R. Kembleton

EUROfusion Programme Management Unit

Garching, Germany

A. W. Morris

CCFE, UKAEA

Abingdon, UK

E. Patelli

Institute for Risk and Uncertainty, University of Liverpool

Liverpool, UK

H. Zohm

Max-Planck-Institute for Plasma Physics

Garching, Germany

\section{Abstract}

During the pre-conceptual design phase of fusion devices such as the European demonstration fusion power plant (DEMO), systems codes provide a fast evaluation of optimal design points and highlight high impact areas. However, determining or evaluating a design point at such an early stage comes with uncertainties in many of the design parameters. These uncertainties are both associated with the physics as well as the engineering basis of the European DEMO design. The work applies an uncertainty quantification analysis to the 2017 pulsed European DEMO design using the PROCESS systems code. It assumes that DEMO will be built as suggested by the baseline and explores what implications the currently known physics and engineering uncertainties have on the expected performance parameters (net electric output and pulse length), while optimising the fusion gain Q. A more detailed single parameter analysis clearly identifies high impact parameters. This confirms previous investigations as well as revealing new areas that warrant deeper investigation in particular in the technology area.

\section{INTRODUCTION}

The European DEMO concept is currently in its pre-conceptual design phase [1]. As a result, both the physics basis and the technology concepts for DEMO have relatively large uncertainties. Nevertheless, it is essential to evaluate the DEMO design and potential alternatives as rigorously as possible given the ambitious timings of the European Roadmap ${ }^{1}$. This allows us to focus on high impact areas as well as conduct a fair comparison of design alternatives with different technology readiness levels or different levels of confidence in its extrapolation.

Uncertainty quantification (UQ) is an ideal way to evaluate the effect of known uncertainties on the predicted results of a machine. Bustreo et al. [2] have investigated the effect of uncertainties on the cost of electricity on a DEMO-like fusion power plant using the FRESCO code. Other uncertainty quantification software like COSSAN-

\footnotetext{
${ }^{1} \mathrm{https}: / /$ www.euro-fusion.org/eurofusion/roadmap/
} 
$X[3]$ is routinely used for a wide spectrum of uncertainty quantification in engineering problems. The top-level European DEMO baseline designs are determined by the system modelling code PROCESS [4,5]. It optimises a figure of merit (e.g. major radius, pulse length, fusion gain) while fulfilling a set of physics and technology constraints using the constrained optimisation solver VMCON [6]. In a preliminary study, Lux et al. [7] have applied an UQ tool combined with the PROCESS code to the 2015 pulsed European DEMO baseline. Their predictions were positive (nearly $90 \%$ likelihood of achieving its set performance goals), but not comprehensive. This work applies the same tool to the 2017 European baseline design [1] and extends the scope of the work to a more complete set of uncertainties (including engineering parameters). Other work applying a similar technique to the Chinese CFETR design has been carried out by Morris et al. [8] and work on the Indian SST-2 design by Muldrew et al. [9].

In this work, we present a combined approach to UQ based on a multi-parameter Monte-Carlo method together with single parameter studies investigating individual impacts. We describe our method in Section 2, the typical uncertainties in input parameters we expect in our systems modelling approach in Section 3 and the implications of our studies on the European DEMO design point evaluation in Section 4. We discuss our results and conclude in Section 5.

\section{METHOD}

In this section, we describe two complementary approaches to evaluate the effect of current physics and technology uncertainties on predicted future power plant designs. Both use the PROCESS systems code as tool to evaluate an optimal design point.

\subsection{Monte-Carlo Approach to Uncertainty Modelling}

The method described in this section, has already been documented more extensively in [10]. However, here we give a short overview of its key aspects: To efficiently determine the effects of uncertainties in the input parameters of a design point evaluation, we use Monte-Carlo sampling within user specified uncertainty distributions. The currently implemented options are

- Gaussian profile (specify mean and standard deviation);

— Lower half Gaussian profile (specify mean and standard deviation);

— Upper half Gaussian profile (specify mean and standard deviation);

- Flat top profile with relative errors (specify mean and percentage);

— Flat top profile with bounds (specify upper and lower bound).

Correlations currently cannot be modelled and are therefore not taken into account. The uncertainties cannot be specified for parameters used as iteration variables in PROCESS as these are varied within the code to determine a constrained optimal design point [4,5]. The output of the method is then the distribution of optimised design points based on the ensemble of randomly varied input points.

We have taken several steps to ensure that each of the individual design point evaluations are robustly converged and, hence, numerical noise intrinsic to the solver will not dominate the final distribution of output parameters. As the constrained optimisation solver implemented in PROCESS (VMCON [6]) is only a local solver, several measures have been implemented to automatically improve the chances of the constrained optimisation solver finding a valid solution, if one exists. This avoids neglecting valid solutions, in cases where the solver struggles to find them. To ensure reproducibility, a seed for the pseudo-random number generator can be specified by the user. One shortcoming of our current approach is that only feasible solutions are being considered in the results, as genuinely invalid input parameter sets cannot be distinguished from parameter sets where PROCESS simply searched too locally for a solution. However, additional to the measures of improving the search strategy for PROCESS, we check the actually produced output distributions. This gives us confidence that relevant cases are generally being captured.

One should note that each PROCESS run will again perform a constrained optimisation analysis and will vary all iteration variables to find an optimal constrained solution. As a result, the solutions might lean on different limits 
LUX, et al.

with respect to the nominal solution. However, this will have a lesser effect than for single-parameter variations (see next section) [11].

\subsection{Single Parameter evaluations/Sensitivity Analysis}

The Monte-Carlo approach described in the previous section, is designed to evaluate the effect of a large range of uncertainties on a DEMO design point at the same time. However, parameters can be correlated, and this approach can make it difficult to interpret individual effects or highlight high impact parameters. Therefore, it is essential to complement this method with single parameter studies to disentangle the competing influences.

For our single parameter evaluations, we again use the previously described uncertainty tool but investigate all uncertain parameters described in Section 3 individually. The result is a distribution which represents the PROCESS response function to the initial input distribution. As such it is more comprehensive than the simple sensitivity studies performed in [12] which only yield two values, but do not show the wider behaviour of the PROCESS code.

\section{UNCERTAINTIES}

In this section, we summarise a selection of known uncertainties in extrapolating the plasma conditions (Section 3.1) as well as technological parameters (Section 3.2) to fusion power plants. The lists describe the relevant quantity and the assumed uncertainty distributions ${ }^{2}$. Where possible this has been motivated with appropriate references. As we cannot model correlations in our method, no correlations between uncertainties are given, but none are expected tween the two groups of physics and technology parameters.

\subsection{Physics Parameters}

Note that the majority of physics uncertainties has already been described in [7] and only deviations are described more extensively here.

Ad hoc multiplication factor for the density limit lower half Gaussian profile (mean 1.2 and standard deviation $0.1)$ As motivated in [7].

Upper bound on H-factor lower half Gaussian (mean 1.2 and standard deviation 0.1) As motivated in [7].

Core radius in radiation corrected confinement time $\tau_{\mathrm{E}}$ scaling Gaussian distribution (mean 0.6 and standard deviation 0.15)

As motivated in [7].

Thermal He-4 fraction Gaussian distribution (mean 0.1 and std 0.025)

As motivated in [7].

W number density fraction relative to the electron density Gaussian distribution (mean $10^{-4}$ and std $5 \times 10^{-5}$ ) As motivated in [7].

Maximum ratio of the divertor figure of merit $\mathbf{P}_{\text {sep }} \mathbf{B}_{\mathrm{T}} / \mathbf{q}_{95} \mathrm{AR}$ Gaussian distribution (mean $8 \mathrm{MW} \mathrm{T/m}$ and std $1 \mathrm{MWT} / \mathrm{m})$

While a more detailed model predicting the power flow and the temperature on the divertor plates is currently undergoing testing [13], this has not been used in the derivation of the most recent DEMO baseline. Therefore, $\mathrm{P}_{\mathrm{sep}} \mathrm{B}_{\mathrm{T}} / \mathrm{q}_{95} \mathrm{AR}$ has been adopted as a divertor figure of merit. This differs from the divertor figure of merit for the pulsed European DEMO baseline from 2015 and therefore the work presented in [7], where $\mathrm{P}_{\mathrm{sep}} / \mathrm{R}$ was used. The new divertor figure of merit is assuming the tolerable power flow to be hitting a ring of surface $2 \pi R \backslash \lambda_{q}[14]$ and furthermore assumes the power decay length at the outer midplane $\lambda_{q}$ scales with the poloidal gyroradius which is proportional to $\mathrm{B}_{\mathrm{T}} / \mathrm{q}_{95} \mathrm{~A}$ [15]. The uncertainty distribution for this figure of merit is equivalent to the one previously used assuming the DEMO 2017 baseline values of $\mathrm{B}_{\mathrm{T}}=4.89 \mathrm{~T}, \mathrm{q}_{95}=3$ and $\mathrm{A}=3.1$.

Lower bound on L-H-threshold limit Gaussian distribution (mean 1.0 and std 0.25 )

As motivated in [7]. 2 We assume that the central limit theorem is applicable, and the uncertainties are typically well described by a Gaussian
distribution. 
Bootstrap current fraction multiplier Gaussian distribution (mean 1.0 and std 0.1 ) As motivated in [7].

Radiation wall load peaking factor Uniform distribution (lower bound 2.0, upper bound 3.5)

The PROCESS systems code does not calculate the distribution of the radiation on the plasma facing components. It therefore needs an estimate of how high the peak radiation wall load is in comparison to an average distribution where all the radiation is distributed evenly along the plasma facing components. Wenninger et al. [16] have estimated what the peak radiation wall load for the pulsed DEMO 2015 baseline design would be. However, the predictions have large uncertainties which are being dominated by the extrapolation of a possible X-point radiator. Assuming a dominant X-point radiator of $150 \mathrm{MW}$ corresponds to a peaking factor of 3.3. However, current experiments suggest that lower value e.g. $60 \mathrm{MW}$ are more realistic. This leads to lower overall peaking factors. Note that this parameter has not been included in the previously published work [7]. Furthermore, this estimate is only valid, if there is no explicit calculation of the radiation from the scrape-off-layer.

Elongation $\kappa$ Lower half Gaussian distribution (mean 1.85 and std 0.05 )

Wenninger et al. [12] have shown that the elongation has a large impact on the net electric output of a design, when optimising the fusion gain at fixed major radius. However, there are still large uncertainties attributed to the maximally allowed elongation. While the 2015 DEMO baseline assumed $\kappa=1.781$ [12] based on a passive stability study [17], the 2017 baseline assumes 1.848 [1]. The nominal value for ITER is 1.85 and the value of the 2015 European baseline for an advanced steady state DEMO (DEMO2) is 2.016 [12]. Higher elongation values are not expected to be controllable, hence, the choice for a lower half Gaussian distribution.

Further known uncertain physics parameters include the pedestal width and height of both the temperature and density profiles. However, apart from the Greenwald limit on the density pedestal height, these are not yet consistently enforced within PROCESS and therefore an analysis of the implications caused by these uncertainties is postponed for future work. Currently an approach is tested, using a more consistent model consisting of a coupled 1D transport and equilibrium solver [18]. Once this has been sufficiently tested within PROCESS, the transport parameters will come with their own set of uncertainties whose impact will have to be evaluated. The introduction of the transport and equilibrium solver is expected to narrow down the allowed design space and will make an uncertainty evaluation even more crucial.

\subsection{Technological Parameters}

The uncertainties that can be treated in the DEMO design heavily depend on the models that are available in PROCESS. Currently the technology models in PROCESS are a bit less detailed than the physics models. Hence, a less complete treatment of uncertainties will be conducted for the technology parameters. Note that DEMO 2015 baseline used NBI as nominal heating source and 2017 baseline used ECRH.

Wall plug efficiency of the CD system (ECRH) Gaussian distribution (mean 0.4 and std 0.1)

Pamela et al. [19] state that the present wall plug efficiency of ECRH systems is of the order of 22\%, while ECRH systems developed for ITER have an expected efficiency of 55\%. More advanced technologies could in theory reach efficiencies as high as $65 \%$. The DEMO baseline makes relatively conservative assumptions by using 0.4 as its wall plug efficiency.

Current drive efficiency Gaussian distribution (mean $0.3 \times 10^{20} \mathrm{~A} / \mathrm{Wm}^{2}$ and std 0.05 )

The nominal value of the current drive efficiency of the ECRH heating system in in 2017 DEMO baseline design point is $0.3 \times 10^{20} \mathrm{~A} / \mathrm{Wm}^{2}$. This corresponds to the analysis by Zohm et al. [20] for flat density profiles.

Plant thermodynamic efficiency Gaussian distribution (mean 0.37 with std 0.05 )

The value in the 2017 DEMO is 0.375 and the value previously used in [12] is 0.37 . The uncertainty on this value does not represent so much the uncertainty of the conversion efficiency of a specific technology, but more the uncertainty about which technology will finally be chosen.

Fractions of the total blanket/divertor/first wall/shield thermal power required to drive the respective coolant pumps Gaussian distribution (mean 0.08 and std 0.005 )

The pumping power is the largest fraction of recirculated power in the 2017 DEMO baseline design followed by the power consumed by the HCD system. Its efficiency is therefore going to have the highest impact on the recirculated power and therefore the net electric output of DEMO. Therefore, other sources of uncertainties in the recirculated power are currently not being assessed. 
LUX, et al.

Some uncertainties would lead to component failure rather than performance reduction. While the European DEMO design already takes a very conservative approach, it is not possible to estimate the probability of component failures from the uncertainty analysis of systems studies. These have to be carried out by more detailed codes.

A sufficient tritium breeding ration is one of the key performance parameters of DEMO. However, the tritium breeding ratio is currently not typically calculated within PROCESS when optimising a design. Rather the blanket thickness is kept fixed that is assumed to give a sufficient TBR for the DEMO size. Hence, the uncertainty in the tritium breeding ratio cannot be evaluated within this work.

\section{IMPLICATIONS FOR DEMO DESIGN}

In this work, we apply our uncertainty quantification tools to the design of the pulsed European DEMO baseline from $2017^{3}$ [1]. The PROCESS reference file for the baseline has been produced with PROCESS version 1.0.10 (commit 48f7f3ee) which also has been used throughout this work.

There are various options how to evaluate the effect of uncertainties on a given design. In this work, we assume DEMO has been built as detailed in the 2017 baseline and we investigate the effect of the know uncertainties on the predicted performance of DEMO.

\subsection{The effect of uncertainties in the physics parameters}

Figure 1 shows the predicted machine performance (pulse length and net electricity) for the pulsed European DEMO baseline for scenarios that are optimised for their fusion gain Q (see Section 2). The distribution shows the results of the uncertainty analysis while the black diamond shows the result of the nominal baseline assumptions. As discussed in [7], the balance of plant (BoP) is likely to only tolerate variations in net electric output of $+5 \% /-20 \%$. While over performing scenarios can be downgraded, scenarios with a net electric output below 400MW have to be classified as not meeting the DEMO requirements. A further assumption is that the DEMO energy storage system can cope with both $1 \mathrm{hr}$ as well as the nominal $2 \mathrm{hrs}$ pulse length and hence scenarios with more than $1 \mathrm{hr}$ pulse length are also accepted.
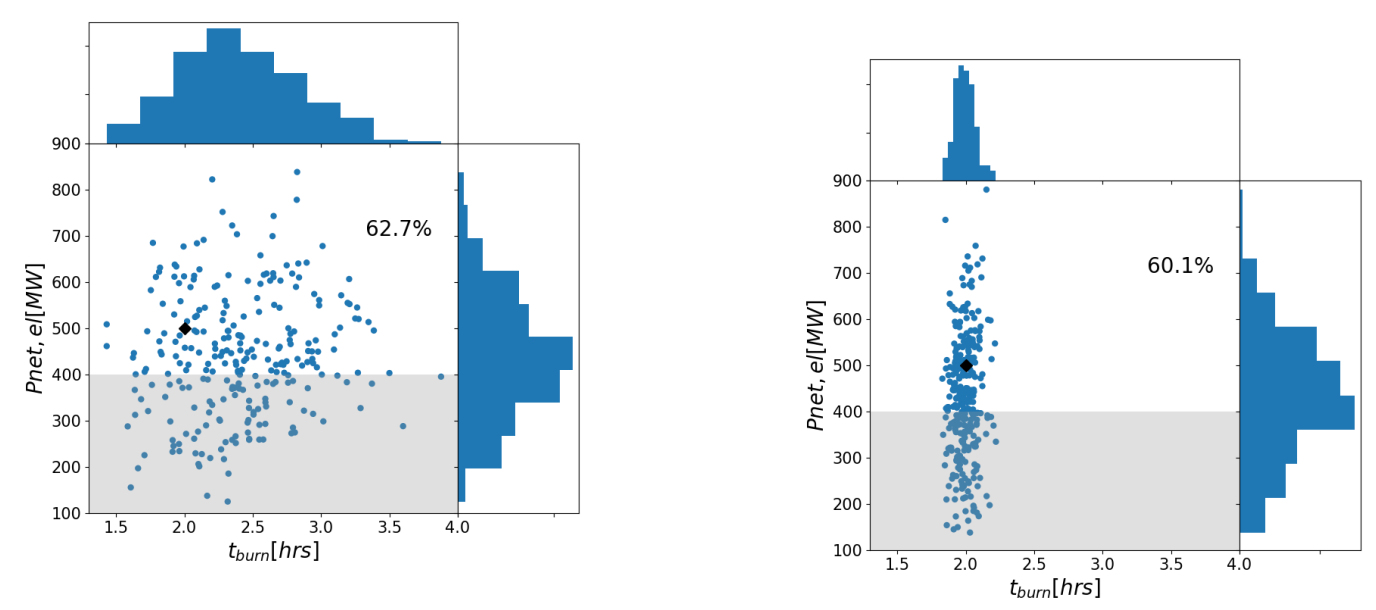

FIG. 1. Predicted machine performance for the 2017 baseline of the pulsed European DEMO design assuming a range of uncertainties in the physics (left side) and technology (right side) parameters. The black diamond indicates the values nominal baseline design.

The left-hand side of Figure 1 shows the results analysing the uncertainties in the physics parameters. With respect to the analysis of the 2015 pulsed DEMO baseline [7], we have extended the list of our uncertain physics parameters by the elongation and the radiation wall load peaking factor and adjusted the uncertainties to the new divertor figure of merit (see Section 3.1). As a result, we find that only $63 \%$ of the scenarios have an acceptable performance, in comparison to the $90 \%$ found by the previous analysis [7]. The elongation has already previously 
found to have a large impact on performance of a power plant [12], while the radiation wall load peaking factor has only been included for completeness in the analysis but does not have a significant impact. Though the dominating driver for the difference with respect to the previous results is the change in the baseline design rather than the extension of the parameter list, as running the same analysis with the old parameter list gives similar numbers.
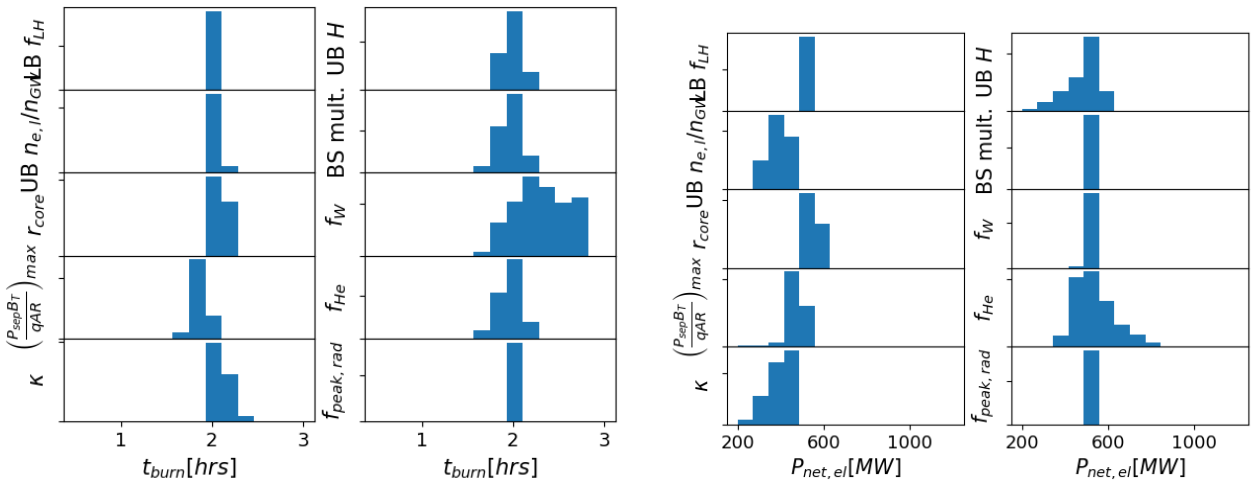

FIG. 2. Single Parameter scans for the uncertain physics parameters left shows the impact on the burn time, while on the right the impact on the net electric output is shown.

Figure 2 shows the individual parameter scans as discussed in Section 2.1. These very clearly illustrate which parameters have a large impact on the prediction of DEMO performance. While some parameters result in a very narrow distribution in performance parameters, others yield a rather wide spread. This shows that the uncertainty in the tungsten fraction $\mathrm{f}_{\mathrm{W}}$ has a significant impact on the predictions of the DEMO pulse length. Furthermore, the uncertainties in the helium fraction $\mathrm{fHe}_{\mathrm{He}}$ and the achievable $\mathrm{H}$-factor cause uncertainties in the predictions of the net electric output as well as the pulse length. Overall the elongation remains the most significant source of uncertainty for the net electric output confirming earlier work [12]. These parameters can therefore be considered to have a high impact on machine design and preference should be given to constraining the uncertainties in their predictions.

\subsection{The effect of uncertainties in the technology parameters}

With respect to the previous analysis [7], not only has the list of physics uncertainties been extended, but also technological uncertainties have been considered. As expected, all technology uncertainties have a significant impact on the net electric output and suggest even the conservative pulsed European DEMO design only has a likelihood of $\sim 60 \%$ in achieving its performance parameters. These results are shown on the right-hand side in Figure 1. The single parameter analysis (not shown) indicates that the plant thermal efficiency has the highest impact on the net electric output of the plant and the current drive efficiency has the highest impact on the burn time.

\section{CONCLUSIONS AND DISCUSSION}

In this work, we analysed the predicted performance of the 2017 baseline design point of the European demonstration power plant DEMO [1] in light of the currently known physics and technology uncertainties. The nominal performance criteria of the baseline DEMO design is a $2 \mathrm{hr}$ pulse length and $500 \mathrm{MW}$ electricity. In this analysis, we assume that DEMO will be build as described in the 2017 baseline design and evaluate the impact of the physics and technology uncertainties described in Section 3 using an uncertainty quantification tool coupled to the PROCESS systems code, which has been used to previously derive the baseline design. We assume that any net electric output of $400 \mathrm{MW}$ or above is still acceptable for the balance of plant and that any pulse length longer than $1 \mathrm{hr}$ is acceptable for the plant storage system. Based on this, we evaluate the likelihood of the current DEMO baseline fulfilling its performance requirements. Please note that this analysis is limited by the models implemented in PROCESS and can therefore neither assess the likelihood of component failures or evaluate the predicted tritium breeding ratio. 
LUX, et al.

The parameter list of the uncertain physics parameters has been extended in this work in respect to the previous investigation of the 2015 pulsed DEMO design. However, in the evaluation of the overall impact the changes in the baseline dominate the reduction in the likelihood of achieving an acceptable performance from about $90 \%$ down to $62 \%$. This reflects that while the nominal baseline parameters have evolved between the baselines, the uncertainty distributions in the analysis have not, possibly not representing the most recent results. The individual parameter analysis reconfirms previous results suggesting that the uncertainties in maximum controllable elongation and impurity fractions are having a significant impact on the machine performance and future work should focus on reducing these.

For the first time, also the impact of uncertainties in the technological input parameters in PROCESS have been investigated. As these mainly cover efficiency parameters of the heating and current drive system as well as the thermal to electricity conversion parameter, they have a significant impact on the machine performance. We find that the overall likelihood of achieving an acceptable performance with respect to the studied technology uncertainties is $60 \%$. Unsurprisingly, the individual parameter analysis suggests a high impact from determining the thermal conversion efficiency well.

\section{ACKNOWLEDGEMENTS}

This work has been (part-) funded by the RCUK Energy Programme [grant number EP/I501045]. To obtain further information on the data and models underlying this paper please contact PublicationsManager@ccfe.ac.uk. Furthermore, this work has been carried out within the framework of the EUROfusion Consortium and has received funding from the Euratom research and training programme 2014-2018 under grant agreement No. 633053. The views and opinions expressed herein do not necessarily reflect those of the European Commission.

\section{REFERENCES}

[1] FEDERICI, G. et al., "DEMO design activity in Europe: Progress and updates”, Fus. Eng. \& Des. 136 (2018) 729-741

[2] BUSTREO, C., BOLZONELlA, T., ZOLLINO, G., "The Monte-Carlo approach to the economics of a DEMO-like power plant”, Fus. Eng. \& Des. 98 (Sup. C) (2015) 2108-2111

[3] PATELLI, E., "COSSAN: A Multidisciplinary Software Suite for Uncertainty Quantification and Risk Management”, Handbook of Uncertainty Quantification, Springer International Publishing, Cham (2016)

[4] KOVARI, M. et al., "PROCESS: A systems code for fusion power plants - Part 1: Physics", Fus. Eng. \& Des. 8912 (2014), 3054-3069

[5] KOVARI, M. et al., "PROCESS: A systems code for fusion power plants - Part 2: Engineering", Fus. Eng. \& Des. 104 (2016), 9-20

[6] CRANE, R. L., HillSTROM, K. E., MiNKOFF, M., "Solution of the General Nonlinear Programming Problem with Subroutine VMCON", Argonne National Laboratory Report ANL-80-64 (1980)

[7] LUX, H., et al., "Uncertainties in power plant design evaluations”, Fus. Eng. \& Des. 123, (2017), 63-

66

[8] MORRIS, J. et al. "Validation and sensitivity of CFETR design using EU systems codes", Fus. Eng. \& Des. (2019), accepted

[9] MULDREW, S. et al, "Uncertainty analysis of an SST-2 fusion reactor design", Fus. Eng. \& Des. (2019), available online

[10] KEMP, R. et al., PMI 7.1.2 Report on systems code activites by CCFE in 2014, EUROfusion report, EFDA_D_2_M94N2 (2014)

[11] LIPSEY, R.G., LANCASTER, K., "The General Theory of Second Best", The Review of Economic Studies, Vol. 24, No. 1 (1956-57), pp.11-32

[12] WENNINGER, R., et al. "The physics and technology basis entering the European system code studies for DEMO”, Nucl. Fus. 571 (2017), 016011

[13] KOVAR, M., KALLENBACH, A., SICCINIO, M., "A one dimensional scrape-off layer model in the reactor systems code 'PROCESS'”, submitted to Fus. Eng. Des. (2019)

[14] ZOHM, H., et al., “A stepladder approach to a tokamak fusion power plant”, Nucl. Fus. 578 (2017) 086002

[15] EICH, T., et al., "Inter-ELM power decay length for JET and ASDEX Upgrade: Measurements and comparison with heuristic drift based model”, Phys. Rev. Lett. 107 (2011) 215001

[16] WENNINGER, R., et al., "The DEMO wall load challenge”, Nucl. Fus. 574 (2017) 046002 
[17] WENNINGER, R. et al., "Advances in the physics basis for the European DEMO design”, Nucl. Fus. 556 (2015) 063003

[18] ELLIS, K., et al. "The next step in system modelling: The integration of a simple 1D transport and equilibrium solver”, EPS conference, Prague (2018)

[19] PAMELA, J., et al., "Efficiency and Availability driven R\&D issues for DEMO”, Fus. Eng. \& Des. 84 2-6 (2009), 194-204

[20] ZOHM, H., et al. "Assesment for H\&CD System Capabilities for DEMO”, 40"th EPS conference on Controlled fusion and plasma physics, Helsinki, Finnland (2013) 